\title{
Three Decades of Distance Education Astronomy at Athabasca University
}

Martin Connors ${ }^{1,2,3 *}$, Farook Al-Shamali ${ }^{1}$, Ken Muntikwa ${ }^{1}$, Ian Schofield ${ }^{1,2}$, Christy Bredson ${ }^{1}$

\begin{abstract}
The first Astronomy course at Athabasca University was offered in 1989 as a correspondence course, using innovative DOS software on the remote students' home computers. A Sciencestream course, it simulated astronomy research and offered laboratory credit at freshman level. The success of this course led to a qualitative astronomy course being offered in the early 1990s, based on a commercially-available course package (including videos) supplemented by practical activities. It also included an essay in which students critique aspects of astronomy in popular culture, based on what they learned in the course. Both courses were popular, but enrollment has plateaued. For more senior students, we developed the possibility to do research projects, which also met a need for senior credit for program students in the B.Sc. We now offer two complementary courses in planetary science, one from an astronomy/physics perspective, and one focusing on planetary geology. Although distance education has come to be more accepted in recent times, and moved to the internet, transitioning our materials to being fully web courses has been challenging. Recent success in transitioning Physics online courses to use of open textbooks suggests that this may be possible in Astronomy as well. We also hope to integrate our online research facilities more into education.

\section{Keywords}

Distance Education - Historical Review - University Level

${ }^{1}$ Centre for Science, Athabasca University, Athabasca AB, Canada

${ }^{2}$ Athabasca University Observatories, Athabasca University, Athabasca AB, Canada

${ }^{3}$ Department of Physics and Astronomy, University of Western Ontario, London ON, Canada

*Corresponding author: martinc@athabascau.ca
\end{abstract}

\section{Introduction}

Distance education may be broadly regarded as presentation of an educational curriculum through materials for study by students who do not physically attend the presenting institution. In recent times the term has become essentially synonymous with "online learning" since that is now a preferred mode of presentation. Generally, we will refer to the term as learning for credentials (formal academic credit), while the more general term "online courses" may include noncredential offerings such as "Massively Open Online Courses" or MOOCs.

Athabasca University (AU) was established in 1970, and thus is approaching its fiftieth year. From early on, it specialized in distance education, at that time mainly taking the form of "correspondence courses". A package of printed materials was mailed out, usually consisting of a textbook and a customized study guide prepared in-house to lead students through the course material. In most cases, students mailed in completed exercises for marking throughout the course, and graded coursework was mailed back to them. Credible exam results 
were guaranteed through use of invigilation centers, which are formal sites set up in AU offices at various locations in Alberta, or in cooperating institutions for exam writing. More creative, but credible, invigilation services can be set up through special arrangement (an often-used example is Royal Canadian Mounted Police, i.e. RCMP or Mountie, outposts in isolated communities). A combination of good quality course materials following curricula similar to those at on-campus institutions, credibility of testing methods, and efforts made to coordinate course credential recognition, led to wide acceptance of AU courses for transfer to other institutions. This is possible not only in Canada, where AU has a large presence in the distant province of Ontario (most populous in Canada), but also in the Unites States, where it is accredited by the Middle States Commission on Higher Education (2018).

A more unique aspect of $\mathrm{AU}$ was the use of "telephone tutors", whose task is to keep in touch with students on a continuous basis to assure, and help with, progress through the course materials. The normal period for course completion is six months for a standard three-credit course, with the possibility to buy extended support to prolong this slightly. This model of home study with a deadline, but with the student having the choice of how quickly to progress, is referred to as "unpaced" or "asynchronous" study, and prevails to this day in core academic disciplines. Some professional faculties, such as Business, have had a large degree of independence, and have modified their methodologies to emphasize a model in which home study students form cohorts and proceed on a common schedule, which is referred to as "paced" or "synchronous" study. These faculties have also moved to models involving "call centers" in which there is no direct interaction of students with a dedicated tutor. The implications of such changes will be briefly discussed at the end of this article.

Well into Athabasca University's existence, an expansion of its course offerings led to development of its first astronomy course, SCIE 280 Introduction to Astronomy and Astrophysics (SCIE being a general Science designation) in 1989 (Hube 1998). This course was developed by Tony Willis, a radio astronomer, and featured a DOS program developed in-house to simulate planetary motions and even proper motion of stars. Ironically in light of what is narrated below, the dominance of DOS and simple methods led to ease of use of the laboratory exercises. Measurements in most of them were made using a ruler on a printout from a dot matrix printer. As a historical note, such printouts were on paper, and a dot matrix referred to the printhead, which pushed pins chosen in small rectangular matrix to force an ink ribbon into contact with the paper. By this method, rough characters could be printed, but images could also be composited with relatively high resolution. Having a relatively challenging set of exercises involving measurement of data, SCIE 280 was classified as a "laboratory science" course, able to be used for laboratory credit in the B.Sc. degree also introduced in the late 1980s, and usually transferring to other institutions as equivalent to their Science-stream courses. As will be detailed below, other courses developed after SCIE 280, and astronomy as a discipline at Athabasca University is now in its thirtieth year. Space-related research takes place at AU (Hube 1998) and is concentrated in planetary science (e.g. Hildebrand et al. 1995; Connors et al. 2011; Wiegert et al. 2017 and space physics (e.g. Connors et al. 2016). Primarily for research purposes but widely used in education, Athabasca University hosts a Skynet $0.4 \mathrm{~m}$ aperture online telescope at its in-town headquarters campus, and a University of North Carolina $0.45 \mathrm{~m}$ telescope at its remote site (Schofield and Connors 2019).

About halfway through the nearly three decades in which AU has offered astronomy courses, a detailed survey was published (Connors et al. 2003), including enrollment analysis. This article will update on progress and challenges to date, and prospects for the future, both at $\mathrm{AU}$ and for astronomy distance education in general.

\section{Developments in Courses}

As noted by Connors et al. (2003), the original course, SCIE 280, was relabelled ASTR 200, while retaining the same course name, in 1995. At this time, a textbook which went out of print was re- 
placed by the Universe text (now Freedman et al. 2014), and this course continues to use that series of textbooks. Modifications to the lab exercises (Connors 1992) were incorporated into the new course, and then-new exercises based on CLEA (Marschall 2000, 1998) were introduced, as well as other attempts at electronic labs (Connors et al. 2003). Ironically, it was found that new developments in computer technology, while quite powerful in making the student lab experience more meaningful and more congruent with actual observational technique, were challenging to implement due to changes of operating systems and computer output methods. A further irony is that when the new course, ASTR 205, was introduced as a non-sciencestream course in 1996, it had a higher course number. Apparently perceiving that lower-numbered courses would be the lowest level, many students enrolled in ASTR 200 who actually should have gone into ASTR 205, with resulting lack of success in a course for which they were not prepared. Explanations on course websites seemed ineffective in combatting this perception, so that finally the step of renumbering ASTR 200 to ASTR 210 took place approximately in 2005 . This seemed to solve the student streaming problem. A lesson is that course numbers matter, especially when viewed in relative isolation on syllabus webpages. As of this writing, however, ASTR 210 is closed and under revision, as the development of new laboratory exercises, always a challenge, has been brought to a virtual standstill by technical issues.

ASTR 205, Universe: The Ultimate Frontier, remains open as our only current freshman course. It remains little changed from the description in Connors et al. (2003). A long course closure in 2017 was needed to convert the course to an online textbook (now Seeds and Backman 2013). The course had been converted to the Moodle learning management system several years ago, recently upgraded to the current software release. It features online testing and assignment submission via Moodle, and invigilated online exams (usually taken in a testing center but on a computer screen: exams are $100 \%$ multiple choice and autograded). Two unique and useful aspects of the course remain: one of the assignments is an essay, near the end of the course in which students critique popular culture item in terms of science learned in the course; and some easy but meaningful observational exercises. Part of the observation is of spectra with a viewer, a small grating which is now the only piece of course material mailed to students. The large enrollment growth cited in Connors et al. (2003) did not persist, and enrollments leveled out at approximately 150 per year in the mid-2000s. This course has also continuously featured videos related to the course materials, initially broadcast on the former provincial educational TV named ACCESS, now privatized. After a period in which videos were loaned by AU's distance education library as tapes or DVDs, they are now streamed. We are unsure to what extent the video materials are used by students. Some of them clearly do view them and find them useful, as we sometimes get questions or comments about them. In general the videos are supplemental to the course textbook and not essential for course success.

GEOL 415, Earth's Origin and Early Evolution, has continued to be offered, but was modified to no longer use a large selection of textbooks, mostly on meteoritics, but instead Moons and Planets (Hartmann 2005). The complementary new course ASTR 310, Planetary Science, was introduced about five years ago, using the same textbook. The courses differ in emphasis, the Geology course stresses concepts from that field, while the Astronomy course emphasizes physics and astronomy. These differences are also enforced through the in-house developed study guides and exercises, and the pre-requisites. Although the number of annual enrollments is modest, these courses play an important role in meeting the demand for senior science courses both by visiting students and by those in our programs.

The lack of senior science credits has led us to offer "project" courses in fields such as astronomy, physics, mathematics, and geology that are relevant to this discussion. We also have the option to label ASTR projects under the general rubric of Science (SCIE), and in principle we could also use the Computing (COMP) designation. We have had steady although small enrollment in, for example, ASTR 
495 and 496, Astronomy and Astrophysics Projects. We have had cases of students doing both of these and then having to choose a further project in another subject area such as physics, with a slight change in emphasis. Some projects are of high enough caliber to merit publication: one led to publication and educational/outreach impact (Noshin et al. 2018, weblink). The general rules that keep project courses of high caliber are that prerequisites are enforced to ensure that incoming students are capable of senior level research work (often relatively independently), and that original research must be done. In the 495 level project courses, it is allowed to mainly do library and online research in original sources, usually as a preparatory study intended to lead to original research work. At the 496 level, completely original work must be done, within the period of the course (although that might include analysis of data taken earlier). In fields such as physics and geology, we often have students presenting research which is related to their full-time careers. In astronomy, this is rare, but sometimes the projects are related to amateur astronomy activities.

\section{Outlook}

Connors et al. (2003) was written at a rather optimistic time when it seemed that Athabasca University could go on to be a leader in the transformation of distance education to online learning. The subsequent decade or slightly more was marred by the university losing viable executive guidance, with consequential negative impacts on the ability of faculty to lead in the field. For example, innovations in online astronomy education in 2018 seem to be coming mostly from the nearby University of Alberta, with its ASTRO 101: Black Holes MOOC, despite the fact that this institution has no special mandate to do distance education. On the other hand, some other institutions have introduced astronomy online courses that did not seem to catch on (e.g. Western University), showing that doing distance education is not as easy as it may look.

As a result, Athabasca University offerings may have stagnated into some reflection of the state of astronomy teaching about the time of the previous article (2003). One overall trend since has been that 3-credit courses covering the entire large field of "astronomy and astrophysics" are no longer favored. There is an increasing tendency to offer a total of six credits at freshman level. For example, the "Universe" (Freedman et al. 2014) textbook is now offered as two "splits". Since a home study student getting an 800 page book by mail or courier can be rather intimidated, it likely is better in many ways to offer split courses, especially in the Science stream where there are also lab exercises to be done. As noted, reliance on outside textbooks can also lead to situations where courses must close to make revisions. For this and to assist with lowering cost to students (at least in principle), we have experimented with open textbooks. The initial reaction to this conversion in physics has been favorable (e.g. Daigle, 2018 weblink). We are currently evaluating Openstax Astronomy to see where it may fit in our subject area. Hopefully, a cost advantage could be implemented, effectively lowering our course fees to benefit more students. However, even without this, de facto control of the revision cycle, as given by an open textbook, is important in distance education, since in our experience course modifications are difficult to make, and associated course closures are costly for us and detrimental to students.

Our way forward in distance education likely lies in adoption of open materials, updating of materials to use appropriate web technologies and data sources, and splitting of courses into at minimum 3 -credit units (smaller course modules are also possible and being considered). Our courses are now online, and well organized under Moodle. This mechanical aspect being under control, we may be able to explore optimum pedagogy. The use of personalized tutoring was a hallmark of $\mathrm{AU}$, but the old model of telephone support at certain fixed hours no longer meets modern lifestyles. More and more interaction with students is by email. The merits of the tutor system itself are being discussed. Our experience is that some students put heavy demands on tutors while others do not, and some even express a wish to work without such support. The merits of call centers hopefully will be care- 
fully evaluated and weighed versus the tutor system, before possibly irreversible changes to one of the distinguishing characteristics of AU distance education.

Although to some extent it is already happening in project courses, one could also wish for enhanced use of our advanced research facilities in student education at all levels. We are exploring having an M.Sc. degree in Science, although progress has been frustratingly slow.

\section{Final words}

It is appropriate to close in paralleling the recent AU experience and new optimism with a quote from Alighieri in 1321, "e quindi uscimmo a revider le stelle", leaving the meaning to be sought as an exercise for the reader (see Figure 1).

\section{References}

Connors, M. (1992). Distance Education in Teaching Introductory Astronomy. Journal of the Royal Astronomical Society of Canada, 86:59.

Connors, M., Douglas, K. A., and Lyder, D. A. (2003). A Decade's Progress in Distance Education Astronomy. Journal of the Royal Astronomical Society of Canada, 97:218.

Connors, M., Schofield, I., Reiter, K., Chi, P. J., Rowe, K. M., and Russell, C. T. (2016). The AUTUMNX magnetometer meridian chain in Québec, Canada. Earth, Planets and Space, 68(1):2.

Connors, M., Wiegert, P., and Veillet, C. (2011). Earth's Trojan asteroid. Nature, 475(7357):481483.

Freedman, R., Geller, R., and Kaufmann, W. J. (2014). Universe: The solar system. Macmillan.

Hartmann, W. K. (2005). Moons and Planets. Belmont, Calif.; Brooks.

Hildebrand, A. R., Pilkington, M., Connors, M., Ortiz-Aleman, C., and Chavez, R. E. (1995). Size and structure of the Chicxulub crater revealed by horizontal gravity gradients and cenotes. Nature, 376(6539):415-417.

Hube, D. P. (1998). Astrophysics At The University of Alberta. Journal of the Royal Astronomical Society of Canada, 92:12.

Marschall, L. A. (1998). Bringing the Universe into the Laboratory-Project CLEA: Contemporary Laboratory Exercises in Astronomy. In International Astronomical Union Colloquium, volume 162, pages 79-87. Cambridge University Press.

Marschall, L. A. (2000). The Universe on a desktop: observational astronomy simulations in the instructional laboratory. Publications of the Astronomical Society of Australia, 17(2):129-132.

Noshin, A., Dang, H., Sewell, G., Funk, K., Vaiji, K., and Shahid, T. (2018). Introduction to Photometry. JRASC, 112(3):116.

Schofield, I. and Connors, M. (2019). Scanning the Auroral Skies: The Athabasca University Robotic Telescope. RTSRE Proceedings, 2(1).

Seeds, M. A. and Backman, D. (2013). Horizons: Exploring the universe. Nelson Education.

Wiegert, P., Connors, M., and Veillet, C. (2017). A retrograde co-orbital asteroid of Jupiter. Nature, 543(7647):687-689. 
Three Decades of Distance Education Astronomy at Athabasca University - 6/6

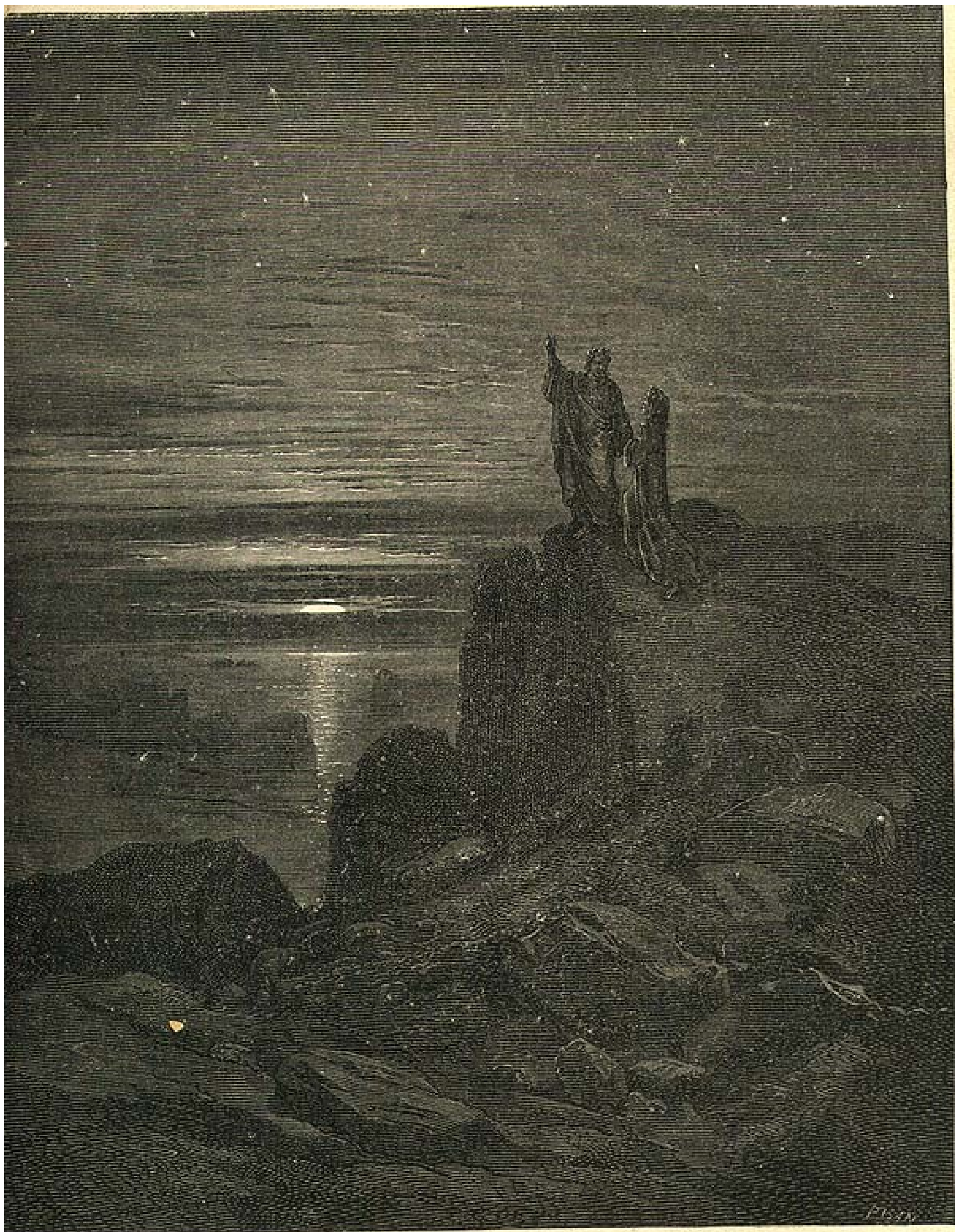

E QUINDt USCIsMo A RIVEDER LE STELLE.

Isrzavo, e. XXXIV, v. 139

Figure 1. Gustav Doré, 1857. A riveder le stelle. 